\title{
Oncogenic circDHTKD1 promotes tumor growth and metastasis of oral squamous cell carcinoma in vitro and in vivo via upregulating miR-326-mediated GAB1
}

\author{
Zhuangzhi $\mathrm{Wu}^{1 \oplus \otimes}$, Xiaoning $\mathrm{He}^{1 \oplus}$, and Siqi Chen ${ }^{1 \oplus}$
}

${ }^{1}$ Department of Stomatology, The Second Affiliated Hospital of Hainan Medical University, Hainan, China

\begin{abstract}
Circular RNAs (circRNAs) have been extensively elucidated with regard to their significant implications in oral squamous cell carcinoma (OSCC). This study performed the functional investigation of circRNA dehydrogenase E1 and transketolase domain containing 1 (circDHTKD1) in OSCC. RNA expression levels of different molecules were measured via quantitative real-time polymerase chain reaction (qRT-PCR). Cellular behaviors were detected by 3-(4, 5-dimethylthiazol-2-y1)-2,5-diphenyl tetrazolium bromide (MTT) for cell viability, colony formation assay for clonal capacity, flow cytometry for cell apoptosis, wound healing assay for migration, and transwell assay for migration/invasion. Western blot was used for analyzing protein expression. RNA pull-down and dual-luciferase reporter assays were applied to assess the binding between targets. A xenograft tumor model was established in nude mice for in vivo experiments. Our expression analysis revealed that circDHTKD1 was upregulated in OSCC tissues and cells. circDHTKD1 knockdown was shown to impede OSCC cell growth and metastasis but motivate apoptosis. Additionally, circDHTKD1 served as a microRNA-326 (miR-326) sponge and the function of circDHTKD1 was achieved by sponging miR-326 in OSCC cells. Also, miR-326 inhibited OSCC development via targeting GRB2-associated-binding protein 1 (GAB1). circDHTKD1 could sponge miR-326 to alter GAB1 expression. Furthermore, circDHTKD1 contributed to OSCC progression in vivo via the miR-326/GAB1 axis. These data disclosed a specific circDHTKD1/miR-326/GAB1 signal axis in governing the malignant progression of OSCC, showing the considerable possibility of circDHTKD1 as a predictive and therapeutic target for clinical diagnosis and treatment of OSCC.
\end{abstract}

Key words: circDHTKD1; Oral squamous cell carcinoma; miR-326; GAB1

\section{Introduction}

Oral cancer has become an alarming global matter as the 11th most common carcinoma and oral squamous cell carcinoma (OSCC) accounts for approximately $90 \%$ of cases of oral cancers (1). OSCC is closely associated with unhealthy lifestyle habits, such as smoking and excessive alcohol intake (2). The primary reason for mortality and relapse of OSCC is the regional or distant metastasis in many parts of the body, thus causing the poor prognosis for OSCC patients (3-5). Hence, it is quite necessary to untangle the tumorigenic mechanism for further target exploration.

Circular RNAs (circRNAs) and microRNAs (miRNAs) are two different sub-families of noncoding RNAs (ncRNAs) that have important regulation in the development of OSCC $(6,7)$. Because of the covalently closed-loop structures by backsplicing, circRNAs are highly stable and more conserved than linear RNAs (8). circRNAs can function as the sponges of miRNAs to influence the interaction between
miRNAs and $3^{\prime}$-untranslated regions ( $3^{\prime}$-UTRs) of target mRNAs to form the classical circRNA/miRNA/mRNA network in a variety of cancers (9-11).

Gao et al. (12) reported that circ-PKD2 impedes the carcinogenesis of OSCC via regulating the miR-204-3p/ APC2 axis. Zhu et al. (13) reported that circRNA_100533 targets the miR-933/GNAS axis to inhibit the malignancy of OSCC. circPVT1 has been identified as a proliferationpromoting factor in OSCC via the regulation of STAT3 by sponging miR-125b (14). A previous study has indicated that circRNA dehydrogenase $\mathrm{E} 1$ and transketolase domain containing 1 (circDHTKD1) is upregulated in OSCC tissues (15), and our preliminary quantitative real-time polymerase chain reaction (qRT-PCR) detection also confirmed its upregulation in OSCC samples. However, its specific function in the progression of OSCC is still unclear. Tao et al. (16) reported that microRNA-326 (miR-326) is a tumorinhibitory factor in OSCC by targeting MTA2. In addition, 
knockdown of GRB2-associated-binding protein 1 (GAB1) blocked cell proliferation, migration, and invasion in OSCC cells (17).

By making a hypothesis that circDHTKD1 can target miR-326 to regulate GAB1, we then designed the explorative experiments to prove it. The function and regulatory mechanism of circDHTKD1 in OSCC are two key points of this study, which may have an important meaning for the circDHTKD1 research in cancers.

\section{Material and Methods}

\section{Patients and tissue specimens}

OSCC patients $(n=56)$ were subjected to surgical treatment at The Second Affiliated Hospital of Hainan Medical University, before which they had received no other therapies. Fifty-six paired OSCC tissues and matched normal adjacent tissues were collected after resection and then stored at $-80^{\circ} \mathrm{C}$ for further experimental use. Written informed consent was obtained from all the involved patients. Biological principles of the Helsinki Declaration for research involving human samples were followed and this study was administered according to the ethical guidelines of the Ethical Committee of The Second Affiliated Hospital of Hainan Medical University.

\section{Cell culture}

Human oral keratinocyte (HOK) cell line was purchased from QCHEN Bio (China) and OSCC cell lines (SCC9 and Cal27) were acquired from American Type Culture Collection (ATCC, USA). All cells were cultivated in a $37^{\circ} \mathrm{C}, 5 \% \mathrm{CO}_{2}$ incubator with a culture solution of Dulbecco's modified Eagle's medium (DMEM; Gibco, USA), $10 \%$ fetal bovine serum (FBS; Gibco), and 1\% penicillin/streptomycin antibiotics (Gibco).

\section{Cell transfection}

Cells were transfected with small interfering RNA targeting circDHTKD1 (si-circDHTKD1) or short hairpin RNA against circDHTKD1 (sh-circDHTKD1, for stable transfection) following the specification of Lipofectamine ${ }^{\mathrm{TM}} 3000$ Reagent (Invitrogen, USA). The miR-326 mimic and inhibitor (miR-326 and anti-miR-326) were used for the overexpression and inhibition of miR-326, and circDHTKD1 or GAB1 overexpression was achieved by the transfection of $\mathrm{pCE}$ RB-Mam-circDHTKD1 (circDHTKD1) or pEXP-RB-MamGAB1 (GAB1) vector. All these oligonucleotides and vectors were bought from RIBOBIO (China), including their respective negative controls (si-NC, sh-NC, miR-NC, antimiR-NC, pCE-RB-Mam, and pEXP-RB-Mam empty vectors).

\section{RNA acquisition and qRT-PCR}

Total RNA was acquired via TRI Reagent (SigmaAldrich, USA) according to the manufacturer's instructions. Reverse transcription was completed by RevertAid RT Reverse Transcription Kit (Thermo Fisher Scientific, USA) and expression was determined using RT-qPCR SYBR Green ROX Kit (Thermo Fisher Scientific). The miRNA quantification was performed by $\operatorname{TaqMan}^{\mathrm{TM}}$ MicroRNA Reverse Transcription Kit and TaqMan MicroRNA Detection Kit (Applied Biosystems, USA) following the manufacturer's instructions. The calculation of relative expression was carried out via the comparative cycle threshold $\left(2^{-\Delta \Delta C t}\right)$ method. The internal gene for circRNA and mRNA was glyceraldehyde-phosphate dehydrogenase (GAPDH) and miRNA levels were normalized by U6. Primers for qRT-PCR were as follows: circDHTKD1, 5'-GACACCTA CATCCCCCTGAA-3' (forward) and 5'-TGAAGAGGG GGTTGATTTTG-3' (reverse); DHTKD1, 5'-CTGCGGT TTGTTGGCATGAA-3' (forward) and 5'-TCATCCGTCTC CTGGCAAAC-3' (reverse); miR-326, 5'-GCCGAGCCT CTGGGCCCTTC-3' (forward) and 5'-CAGTGCAGGGTC CGAGGTAT-3' (reverse); miR-330-5p, 5'-TCGGCAGGT CTCTGGGCCTGTG-3' (forward) and 5'-CGCAGGGTC CGAGGTAT-3' (reverse); miR-338-3p, 5'-TGCGGTCC AGCATCAGTGAT-3' (forward) and 5'-CCAGTGCAGGGT CCGAGGT-3' (reverse); GAB1, 5'-TGGCAGCTCTTTAC AAGCACC-3' (forward) and 5'-TCATGAGCAACAGGTA GTCTTGA-3' (reverse); GAPDH, 5'-TCAAGGCTGAGA ACGGGAAG-3' (forward) and 5'-TCGCCCCACTTGATT TTGGA-3' (reverse); U6, 5'-CTCGCTTCGGCAGCACA TATACTA-3' (forward) and 5'-AAATATGGAACGCTTCAC GA-3' (reverse).

\section{Analysis of stability and localization}

For stability analysis, $5 \mu \mathrm{g}$ total RNA was incubated with $20 \mathrm{U}$ Ribonuclease R (RNase R; Epicentre Technologies, USA) at $37^{\circ} \mathrm{C}$ for $1 \mathrm{~h}$ and $\mathrm{qRT}$-PCR was carried out to assay circDHTKD1 and linear DHTKD1 levels. In addition, SCC9 and Cal27 cells were treated with $2 \mathrm{mg} / \mathrm{mL}$ actinomycin D (Sigma-Aldrich) for $0,4,8,12$, or $24 \mathrm{~h}$ followed by qRT-PCR detection for circDHTKD1 and linear DHTKD1. For the cellular localization, PARIS ${ }^{\mathrm{TM}}$ Kit (Invitrogen) was applied for isolating the nuclear or cytoplasmic RNA and circDHTKD1 was examined by qRT-PCR using GAPDH and $U 6$ as the control groups.

\section{MTT assay}

After seeding SCC9 and Cal27 cells $\left(1 \times 10^{4} /\right.$ well $)$ onto 96-well plates for $24 \mathrm{~h}$, transfection was conducted for $0,12,24$, and 48 h. CyQUANT ${ }^{\mathrm{TM}}$ MTT Cell Viability Assay (Invitrogen) was used for the detection of cell viability. In brief, $10 \mu \mathrm{L}$ MTT [3-(4,5-dimethylthiazol-2-y1)-2, 5-diphenyl tetrazolium bromide] was instilled into each well for $4 \mathrm{~h}$-incubation and the generated formazan was dissolved by adding $100 \mu \mathrm{L}$ SDS-HCl solution. Finally, the absorbance was read under a microplate reader (Thermo Fisher Scientific) at $570 \mathrm{~nm}$.

\section{Colony formation assay}

After transfection for $24 \mathrm{~h}$, cells were plated onto 6-well plates with the initial density of 500 cells/well and cultured 
in DMEM medium containing 10\% FBS under the normal condition for 14 days. At room temperature, the colonies were immersed in methanol (Sigma-Aldrich) for 10-min fixation and in crystal violet (Sigma-Aldrich) for 15-min staining. Three biological and three technical replicates were performed for each group. The images of colonies were obtained by a light microscope (Thermo Fisher Scientific) and all colonies in each well were manually counted with 50 cells as one colony.

\section{Flow cytometry}

Seventy-two hours after cell transfection, Annexin V-FITC/Propidium lodide (PI) Apoptosis Detection Kit (Dojindo, Japan) was used for apoptosis analysis in strict accordance with the manufacturer's instructions. Then, the flow cytometer (BD Biosciences, USA) was used to differentiate the apoptotic cells. Annexin V (+)/PI(-) stained cells were considered as the viable apoptotic cells while Annexin $\mathrm{V}(+) / \mathrm{PI}(+)$ stained cells represented the apoptotic cells. Apoptosis rate was calculated using the ratio of apoptotic cells / total cells $\times 100 \%$.

\section{Wound healing assay}

Transfected SCC9 and Cal27 cells $\left(2 \times 10^{5}\right)$ were inoculated onto 6-well plates to culture for monolayer confluence. Then, two straight scratches were created using a sterile $200 \mu \mathrm{L}$ pipette tip, followed by cell washing with phosphate buffer solution (PBS) and incubation in cell medium without FBS. Images were obtained by a light microscope $(40 \times$, Thermo Fisher Scientific) at 0 and $24 \mathrm{~h}$ and the wound healing rate $(\%)$ is reported as wound width $_{(0 h)}-$ wound width $_{(24 \text { h) }} /$ wound width $_{(0 ~ h)}$.

\section{Transwell assay}

Migration and invasion were analyzed using transwell chambers (Corning Inc., USA) and matrigel (Corning Inc.) pre-coated transwell chambers, respectively. Cells $\left(1 \times 10^{5}\right)$ resuspended in serum-free medium were seeded into the upper chamber, while 10\% FBS + DMEM medium was added into the lower chamber. Twenty-four hours later, cells across the membranes were collected and dyed using $4 \%$ paraformaldehyde and crystal violet (Sigma-Aldrich). Migrated and invaded cells were photographed under an inverted microscope $(100 \times$ magnification, Olympus, Japan) and counted.

\section{Western blot}

To obtain total proteins, cells were lysed in RIPA Buffer (Cell Signaling Technology (CST), USA). BCA Protein Assay Kit (CST) was employed for the determination of protein concentration. According to previous publications $(18,19)$, western blot was carried out using $20 \mu \mathrm{g}$ proteins in each group. Primary antibodies were matrix metalloproteinase 2 (MMP2, CST, \#40994, 1:1000), MMP9 (CST, \#13667, 1:1000), GAB1 (CST, \#3232, 1:1000), and GAPDH (CST, \#5174, 1:1000). Anti-rabbit IgG, HRP-linked antibody (CST, \#7074, 1:2000) was used to combine with primary antibody to form the protein complex, followed by the detection by SignalFire ${ }^{\mathrm{TM}}$ Plus ECL Reagent (CST). Ultimately, the grey analysis of each gene was performed via the Imagelab software version 4.1 (Bio-Rad, USA) and GAPDH acted as the housekeeping gene for other objective genes.

\section{RNA pull-down assay with circDHTKD1 probe}

C-1 magnetic beads (Life Technologies, USA) were pre-coated with circDHTKD1 probe or Oligo probe (RIBOBIO) at room temperature for $2 \mathrm{~h}$. SCC9 and Cal27 cells transfected with vector or circDHTKD1 were lysed and incubated with the probe-coated magnetic beads at $4^{\circ} \mathrm{C}$ overnight and qRT-PCR was used for the expression analysis of circDHTKD1. In addition, cell lysates of SCC9 and Cal27 cells were also incubated with the above probecoated magnetic beads followed by the enrichment detection of different miRNAs (miR-326, miR-330-5p, and $\mathrm{miR}-338-3 p)$.

\section{Dual-luciferase reporter assay}

circDHTKD1 sequence (wild-type, WT) or its mutated sequence (mutant-type, MUT), based on the miR-326 binding sites, was amplified to perform molecular cloning via the basic luciferase gene vector pGL3-control (Promega, USA). The luciferase vector containing circDH TKD1 WT or MUT sequence was termed as circDHTKD1 WT and circDHTKD1 MUT. GAB1 $3^{\prime}$-UTR-WT and GAB1 $3^{\prime}$-UTR-MUT were constructed in the same way. Each plasmid was then transfected into SCC9 and Cal27 cells together with miR-326 or miR-NC. After transfection for $48 \mathrm{~h}$, the firefly and renilla luciferase signals were measured by the dual-luciferase reporter assay system (Promega), according to the manufacturer's guidelines. The ratio of firefly and renilla was used to denote the relative luciferase activity.

\section{In vivo experiment}

To establish the xenograft tumor model, six-week-old male BALB/c nude mice (Vital River Laboratory Animal Technology, China) were subcutaneously injected with SCC9 cells $\left(2 \times 10^{6} / 0.2 \mathrm{~mL}\right.$ PBS $)$ or sh-NC/sh-circDHTKD1 transfected SCC9 cells (6 mice for each group). After cell inoculation, tumor length ( $L$ ) and width (W) were measured by a digital caliper every 5 days, followed by the calculation of tumor volume $\left(\mathrm{L} \times \mathrm{W}^{2} \times 0.5\right)$. Twenty-five days later, those mice were sacrificed by displacing the $60 \%$ air of the cage using the flow rate of $\mathrm{CO}_{2}$. After the dissected tumors were weighed, the total RNA and protein were extracted for qRT-PCR and western blot analyses. Animal care followed the Management and Use Guidelines of Laboratory Animals of the National Institutes of Health (NIH) and this animal assay was approved by the Animal Ethical Committee of The Second Affiliated Hospital of Hainan Medical University. 


\section{Statistical analysis}

SPSS 24.0 (IBM Corp., USA) was used for statistical analysis, and figure production was completed using Graphpad Prism 7 (GraphPad Inc., USA). All data are reported as means $\pm S D$ with three independent experiments of $n=3$. Student's $t$-test and one-way analysis of variance (ANOVA) followed by Tukey's test were adopted for analysis of different groups. $\mathrm{P}<0.05$ was considered to be a significant difference.

\section{Results}

\section{Identification of upregulated circDHTKD1 in OSCC tissues and cells}

circDHTKD1 originates from the exon 2-10 of DHTKD1 gene in the chr10: 12123470-12143180, and our conventional PCR confirmed that the length of circDHTKD1 was $1742 \mathrm{bp}$ as shown in Figure 1A. Through the detection of circDHTKD1 in our collected 56 OSCC samples relative to normal samples, circDHTKD1 was identified to be overexpressed as a dysregulated circRNA (Figure 1B). The clinicopathological characteristics have demonstrated that high expression of circDHTKD1 was associated with the clinical stages and tumor size (Table 1). Also, circDHTKD1 expression was higher in SCC9 and Cal27 cells than in HOK cells (Figure 1C). No difference of circDHTKD1 level was observed but linear DHTKD1 was largely decreased after RNase $\mathrm{R}$ treatment, showing the high stability of circDHTKD1 as a circRNA (Figure 1D and E). Also, a time course with actinomycin $D$ showed that the half-life of circDHTKD1 was longer than linear DHTKD1 (Figure 1F and $\mathrm{G}$ ). The exonic circRNAs are commonly localized in the cytoplasm (20). Through comparing the expression of circDHTKD1 with the levels of cytoplasmic GAPDH and nuclear U6, we identified that circDHTKD1 was principally localized in the cytoplasm not only in normal HOK cells (Figure $1 \mathrm{H}$ ) but also in SCC9 and Cal27 cells (Figure $1 \mathrm{I}$ and J). circDHTKD1 was identified as an upregulated circRNA in OSCC.

\section{Silencing circDHTKD1 suppressed cell growth and metastasis and induced apoptosis of OSCC cells}

Transfection of si-circDHTKD1 was applied for expression knockdown of circDHTKD1 in SCC9 and Cal27 cells. As shown in Figure $2 A$ and $B$, circDHTKD1 was significantly downregulated in the si-circDHTKD1 group compared with the si-NC group but DHTKD1 mRNA expression was unchanged. With the knockdown of circ DHTKD1, cell viability (Figure 2C and D) and clonal ability (Figure 2E) were significantly inhibited while cell apoptosis rate was increased (Figure 2F) in SCC9 and Cal27 cells. By the wound healing assay, we found that cell migratory ability was restrained by the downregulated circDHTKD1 in SCC9 and Cal27 cells (Figure 2G-H). Also, cell migration
A

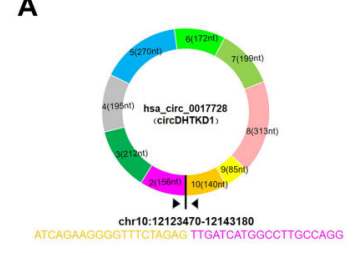

D

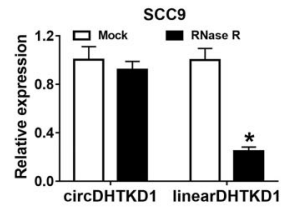

H

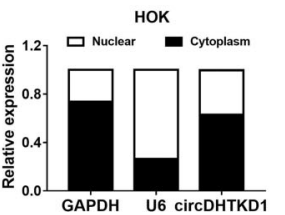

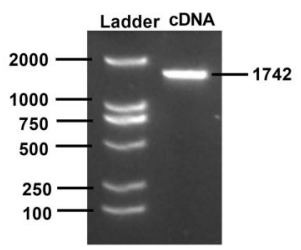

E
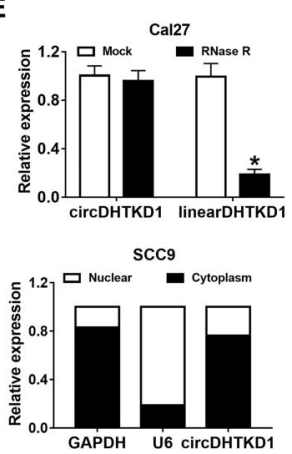

B

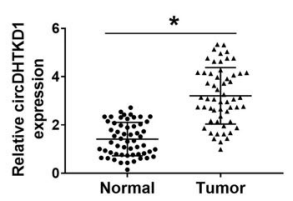

F

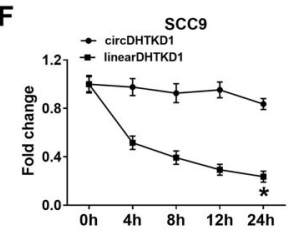

J

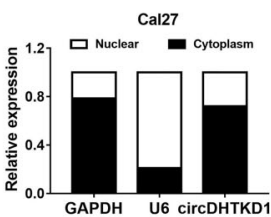

C

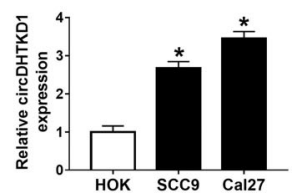

G

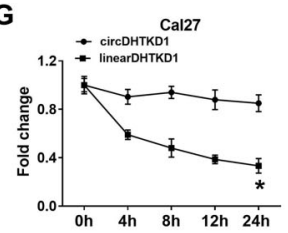

Figure 1. Identification of upregulated circDHTKD1 in oral squamous cell carcinoma (OSCC) tissues and cells. A, Genetic information of circDHTKD1 and the analysis of circDHTKD1 length by conventional PCR. B and C, qRT-PCR was applied for expression of circDHTKD1 in OSCC/normal tissues and OSCC (SCC9 and Cal27) cells/HOK cells. Three biological and three technical replicates were performed. circDHTKD1 and linear DHTKD1 levels were determined using qRT-PCR after RNase R treatment for total RNA (D and E) and actinomycin D treatment for OSCC cells (F and G). Three biological and three technical replicates were performed. $\mathbf{H}-\mathbf{J}$, The detection of circDHTKD1, GAPDH, and U6 was carried out via qRT-PCR in nuclear and cytoplasmic fractions of HOK cells and OSCC cells. Three biological and three technical replicates were performed. Data are reported as means $\pm S D$. * $P<0.05$ (Student's $t$-test). 
Table 1. Correlation between circDHTKD1 expression and clinicopathological characteristics in oral squamous cell carcinoma patients $(n=56)$.

\begin{tabular}{|c|c|c|c|c|}
\hline \multirow[t]{2}{*}{ Clinicopathologic parameters } & \multirow[t]{2}{*}{ Patients } & \multicolumn{2}{|c|}{ circDHTKD1 expression } & \multirow[t]{2}{*}{$P$ value } \\
\hline & & Low $(n=28)$ & High $(n=28)$ & \\
\hline Gender & & & & 0.2801 \\
\hline Male & 32 & 18 & 14 & \\
\hline Female & 24 & 10 & 14 & \\
\hline Age (years) & & & & 0.2646 \\
\hline$\leqslant 60$ & 36 & 20 & 16 & \\
\hline$>60$ & 20 & 8 & 12 & \\
\hline Clinical stage & & & & $0.0145^{*}$ \\
\hline$|-| \mid$ & 23 & 16 & 7 & \\
\hline III-IV & 33 & 12 & 21 & \\
\hline Tumor size $(\mathrm{cm})$ & & & & 0.0533 \\
\hline$<3$ & 21 & 14 & 7 & \\
\hline$\geqslant 3$ & 35 & 14 & 21 & \\
\hline Differentiation & & & & 0.1582 \\
\hline Well/Moderate & 37 & 21 & 16 & \\
\hline Poor & 19 & 7 & 12 & \\
\hline
\end{tabular}

${ }^{*} \mathrm{P}<0.05$, chi-squared test.

(Figure 2l) and invasion (Figure 2J) by transwell assay were reduced after transfection of si-circDHTKD1 compared to si-NC transfection. MMPs can reflect cell invasion and aggressiveness in many tumors $(21,22)$. Our western blot indicated the inhibitory effects of si-circDHTKD1 on MMP2 and MMP9 protein levels compared with the si-NC group (Figure $2 \mathrm{~K}$ and $\mathrm{L}$ ), indicating that circDHTKD1 downregulation blocked cell invasion. Taken together, silencing circDHTKD1 suppressed OSCC cell growth and metastasis but enhanced apoptosis.

circDHTKD1 acted as a molecular sponge of miR-326

To search for the miRNA targets of circDHTKD1, circ DHTKD1 probe was designed and its functional affirmation was performed first. RNA pull-down assay showed that circDHTKD1 probe caught more circDHTKD1 in vector or circDHTKD1 transfection groups than Oligo probe, and circDHTKD1 expression was higher by circDHTKD1 probe following circDHTKD1 overexpression (Figure 3A and B), which suggested that circDHTKD1 probe was successful to capture circDHTKD1. Venn diagram was used to analyze the common miRNAs predicted by starBase (starBase (https//bio.tools), circBank (http//:circbank.cn), and Circlnteractome (http://circinteractome.nia.nih.gov). As Figure 3C describes, three miRNAs (miR-326, miR-330-5p, and miR338-3p) were selected as the potential candidate targets for circDHTKD1. Then, pull-down assay revealed that miR-326 was the most significant miRNA pulled down by circDHTKD1 probe compared with Oligo probe (Figure 3D and $\mathrm{E}$ ), thus the target relation of miR-326 and circDHTKD1 was further confirmed. starBase showed that there were two target regions between miR-326 and circDHTKD1 sequences (Figure 3F). Dual-luciferase reporter assay showed that miR-326 could interact with circDHTKD1 to inhibit the luciferase signal of circDHTKD1 WT plasmid, with no effect on the mutated circDHTKD1 MUT plasmid (Figure 3G and $\mathrm{H}$ ). OSCC tissue samples and cells (SCC9 and Cal27) were found to express miR-326 at a low level, relative to normal tissues and HOK cells (Figure $3 \mathrm{I}$ and $\mathrm{J}$ ). In addition, miR-326 expression was elevated after circDHTKD1 knockdown in SCC9 and Cal27 cells (Figure $3 \mathrm{~K}$ and L). The miR-326 expression was increased perhaps because some miR-326 absorbed by circDHTKD1 was released after circDHTKD1 was knocked-down. Thus, circDHTKD1 had the sponge effect on miR-326 in OSCC cells.

\section{Inhibition of miR-326 partly restored the cancer- inhibitory function of downregulated circDHTKD1 in OSCC cells}

Reversed experiment was performed through the transfection of si-NC, si-circDHTKD1, si-circDHTKD1+ antimiR-NC, and si-circDHTKD1 + anti-miR-326. qRT-PCR demonstrated the large repressive efficiency of anti-miR326 because of its significant neutralization for the sicircDHTKD1-induced miR-326 upregulation (Figure 4A and $B$ ). As the results of miR-326 inhibition, cell growth decline (Figure 4C-E) and apoptosis acceleration (Figure $4 \mathrm{~F}$ ) caused by silence of circDHTKD1 were recovered to a large extent. By conducting the metastasis-related assays (wound healing assay, transwell assay, and western blot), we found that the si-circDHTKD1-mediated inhibitory influences on migration (Figure 4G-I) and invasion (Figure $4 \mathrm{~J}-\mathrm{L}$ ) were rescued via the introduction of miR-326 inhibitor. At least in part, the effects of circDHTKD1 knockdown on OSCC cells were related to miR-326 upregulation. 
A
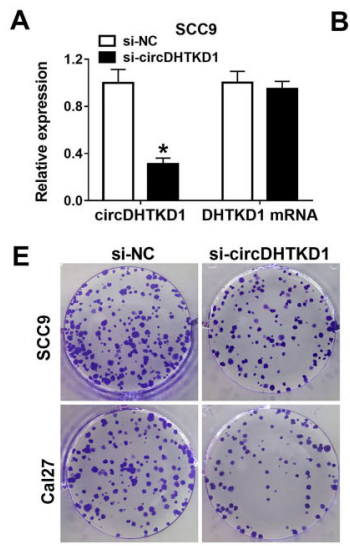

B
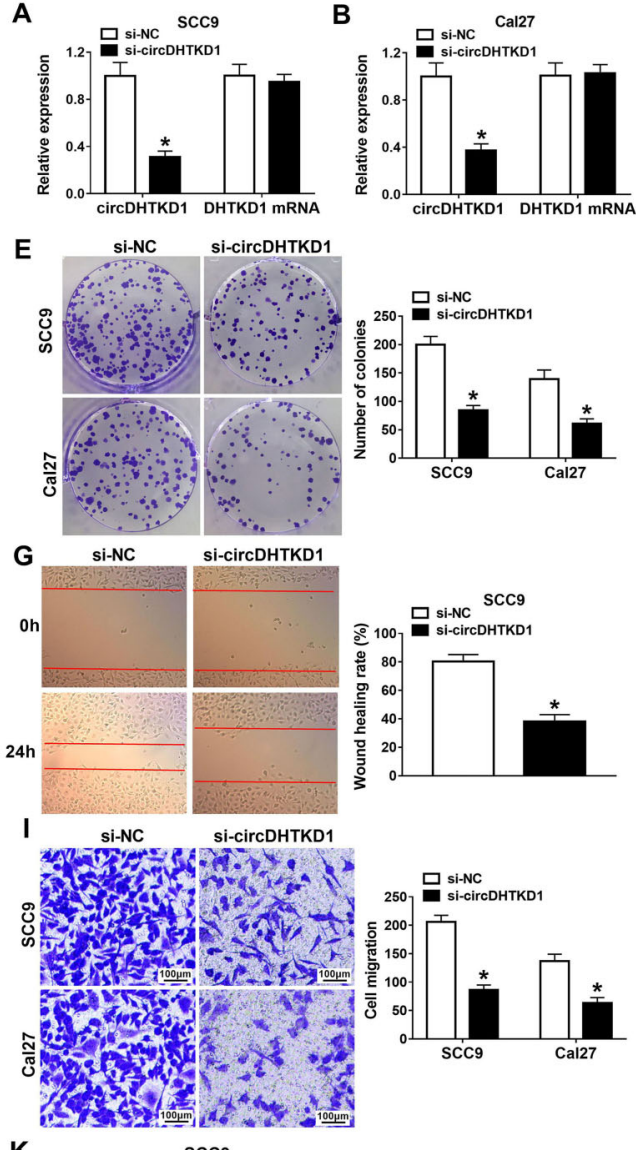

K
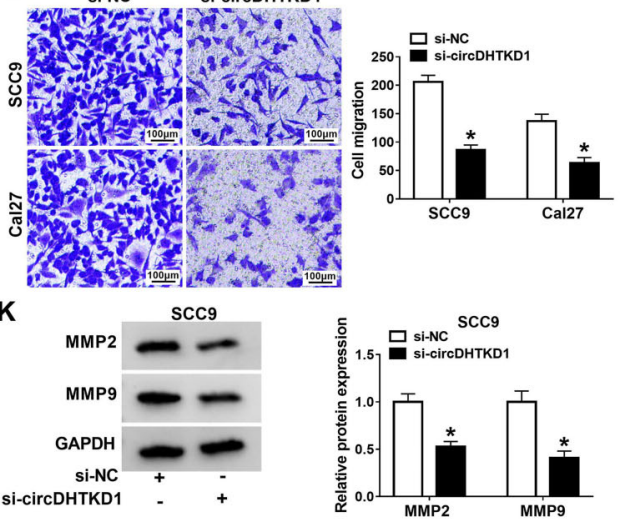

C
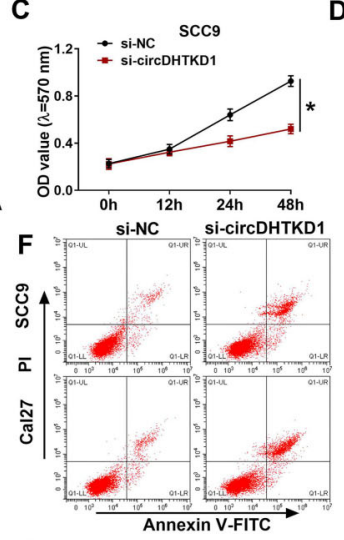

H si-NC si-circDHTKD
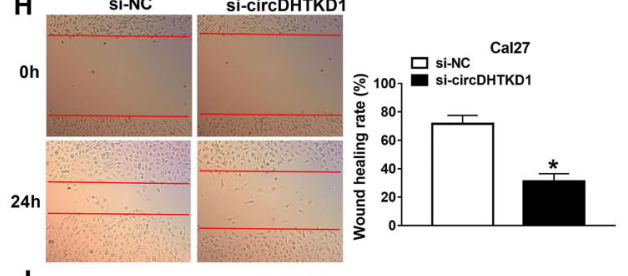

$\mathbf{J}$
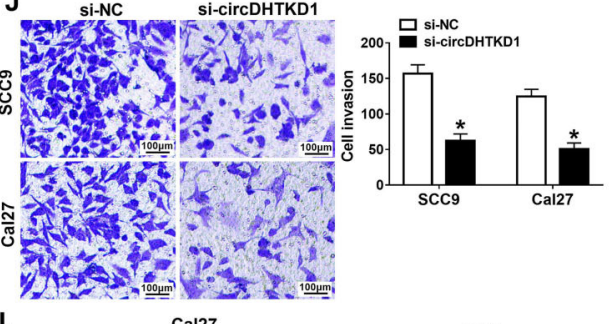
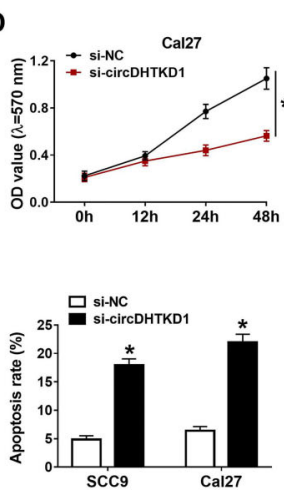

L

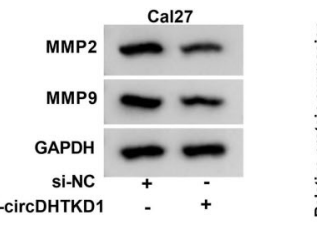

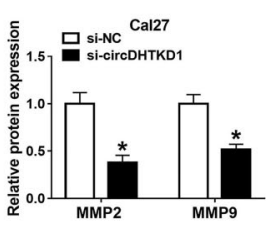

Figure 2. Silencing circDHTKD1 suppressed cell growth and metastasis and induced apoptosis of oral squamous cell carcinoma (OSCC) cells. SCC9 and Cal27 cells were transfected with si-NC (negative control) or si-circDHTKD1. A and B, The levels of circDHTKD1 and DHTKD1 mRNA were assayed by qRT-PCR. MTT and colony formation assays were, respectively, used for analyzing cell viability ( $\mathbf{C}$ and $\mathbf{D})$ and clonal ability $(\mathbf{E})$. F, Apoptosis was measured by flow cytometry. $\mathbf{G}$ and $\mathbf{H}$, Wound healing assay was carried out to assess cell migration ability. Transwell assay was used for analyzing cell migration (I) and invasion (J) (scale bar: $100 \mu \mathrm{m})$. K and L, The protein levels of MMP2 and MMP9 were detected using western blot. Three biological and three technical replicates were performed in each assay. Data are reported as means \pm SD. ${ }^{*} P<0.05$ (Student's $t$-test).

\section{circDHTKD1 regulated GAB1 expression via} interacting with miR-326

After the prediction of starBase, we noticed that the sequence of GAB1 3 -UTR contained the complemental sites for miR-326 (Figure 5A). In the dual-luciferase reporter assay, a decline was observed regarding the luciferase activity of GAB1 3'-UTR-WT but not of GAB1 $3^{\prime}$-UTR-MUT vector by the overexpressed miR-326 (Figure 5B and C), revealing the combination of miR-326 and the $3^{\prime}$-UTR of GAB1. qRT-PCR and western blot showed the high mRNA and protein expression of GAB1 in OSCC tissues (Figure 5D and E) and cells (Figure 5F and $\mathrm{G})$, compared with normal tissues and HOK cells. Additionally, GAB1 mRNA and protein levels were significantly reduced in miR-326-transfected SCC9 and Cal27 cells compared to miR-NC-transfected cells (Figure $5 \mathrm{H}$ and I). More interestingly, si-circDHTKD1 downregulated the mRNA and protein levels of GAB1, which were then reversed by miR-326 inhibitor (Figure 5J and K). Thus, GAB1 was regulated by the circDHTKD1-mediated miR-326. 
A

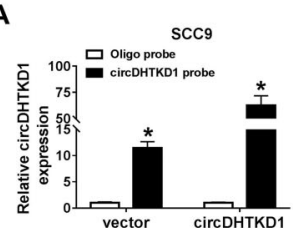

D

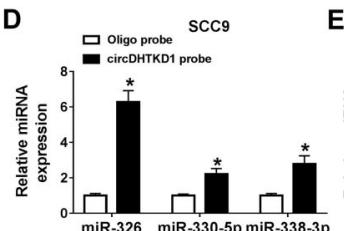

$\mathbf{G}$
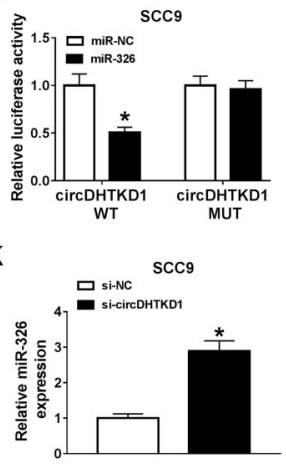

B
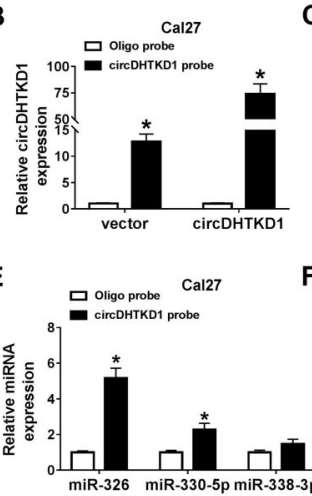

$\mathrm{H}$
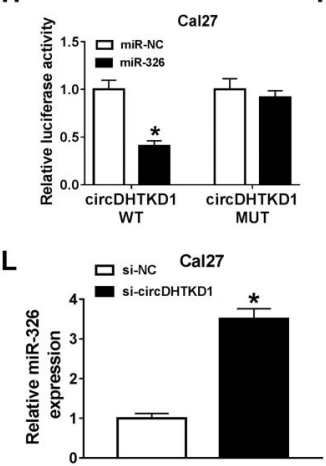

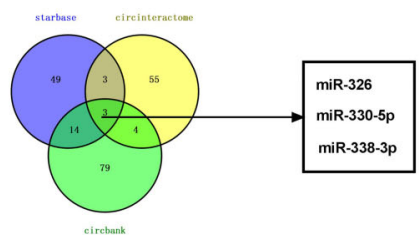

Binding Site of miR-326 on circDHTKD1 chr10:12136105-12136122[+]

circDHTKD1 WT 5' aUGGAGACA--.--GCCCAGAGg3'

$$
\text { miR-326 II gACCUCCUUCCCGGGUCUCC 5' }
$$

circDHTKD1 MUT 5' aGUAGUACA----AUUAGUGAg3'

$J$
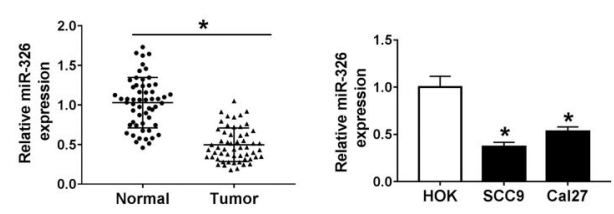

Figure 3. circDHTKD1 acted as a molecular sponge of miR-326. A and B, RNA pull-down assay was performed in SCC9 and Cal27 cells transfected with vector or circDHTKD1 to analyze the action of circDHTKD1 probe. C, The common miRNAs from starBase, circBank, and Circlnteractome were analyzed by Venn diagram. D and E, Different miRNAs levels were examined by qRT-PCR after RNA pull-down with circDHTKD1 probe in SCC9 and Cal27 cells. F, The circDHTKD1 and miR-326 interaction was predicted by starBase. $\mathbf{G}$ and $\mathbf{H}$, The binding of circDHTKD1 with miR-326 was identified by dual-luciferase reporter assay. qRT-PCR was employed to determine the relative expression of miR-326 in oral squamous cell carcinoma tissues (I) and cells $(\mathbf{J})$. $\mathbf{K}$ and $\mathbf{L}$, The effect of si-circDHTKD1 on miR-326 level was analyzed via qRT-PCR using si-NC (negative control) as the control group. Three biological and three technical replicates were performed in each assay. Data are reported as means $\pm S D$. ${ }^{*} \mathrm{P}<0.05$ (Student's $t$-test and one-way ANOVA followed by Tukey's test).

\section{GAB1 downregulation was accountable for miR-326 as a tumor inhibitor in OSCC}

As for the function and mechanism analyses of miR326 in OSCC, SCC9 and Cal27 cells were transfected with miR-NC, miR-326, miR-326 + vector, and miR-326 + GAB1. As shown in Figure 6A and B, GAB1 transfection eliminated the miR-326-induced inhibition of GAB1 protein expression. MTT and colony formation assays showed that miR-326 obstructed cell viability (Figure 6C and D) and colony formation capacity (Figure 6E), whereas these effects were antagonized by overexpressing GAB1. The apoptosis-promoting action in flow cytometry (Figure 6F) and migration inhibition in the wound healing assay (Figure $6 \mathrm{G}$ and $\mathrm{H}$ ) by miR-326 were partially ameliorated after GAB1 was upregulated. In contrast with the miR-326 transfection group, migrated and invaded cells of the miR326 + GAB1 group were significantly increased in SCC9 and Cal27 cells (Figure $6 \mathrm{I}$ and $\mathrm{J}$ ). The same counterbalance of GAB1 for miR-326 was found in the decrease of MMP2 and MMP9 proteins (Figure 6K and L). These findings revealed that miR-326 worked as a tumor inhibitor in OSCC by downregulating GAB1.

\section{circDHTKD1 promoted OSCC progression in vivo by sponging miR-326 to regulate GAB1}

To further validate the potential of circDHTKD1 as a therapeutic biomarker in OSCC, we established the xenograft model through the injection of tumor cells in mice. After 25 days, tumor volume in the sh-circDHTKD1 group was smaller than that in control and sh-NC groups (Figure 7A). Tumor weight was also reduced after the knockdown of circDHTKD1 (Figure 7B and C). qRT-PCR suggested that circDHTKD1 expression was downregulated (Figure 7D) while miR-326 level was increased (Figure 7E) in the sh-circDHTKD1 group compared with control and sh-NC groups. Moreover, downregulation of circDHTKD1 evoked decreased protein expression of GAB1, MMP2, and MMP9 in tumors (Figure 7F). Thus, 
A

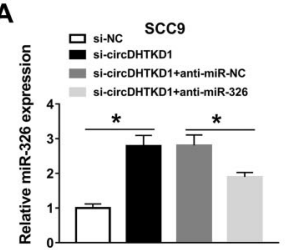

E

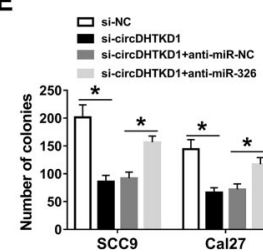

I

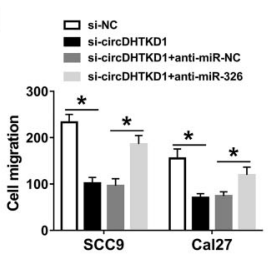

$\mathbf{L}$

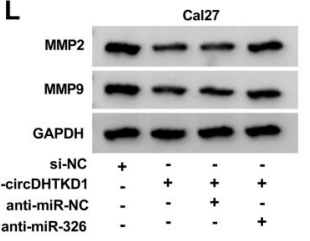

B

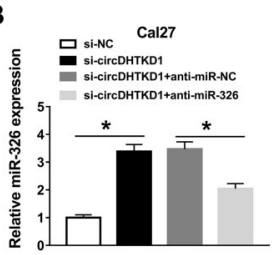

$\mathbf{F}$
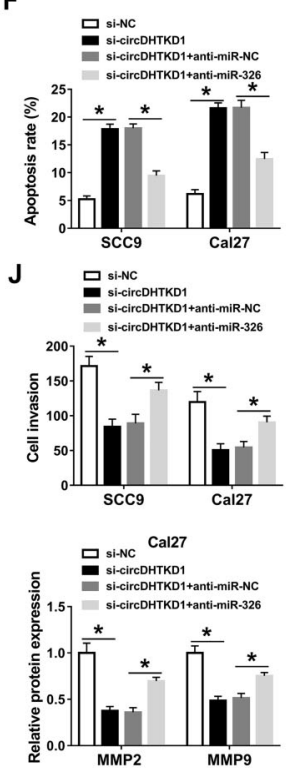

C

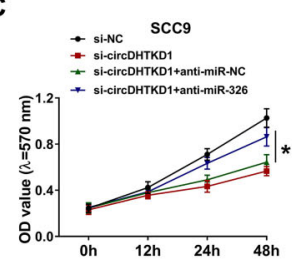

G
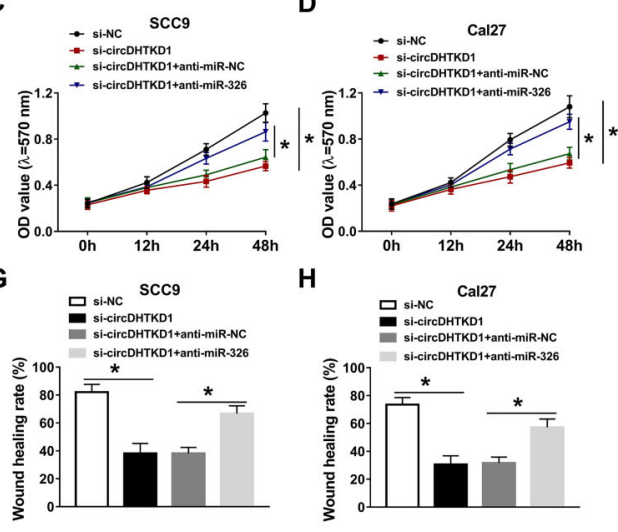

H

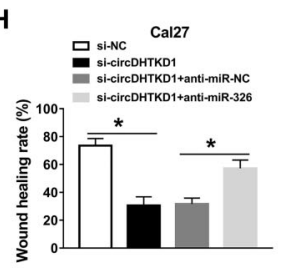

K
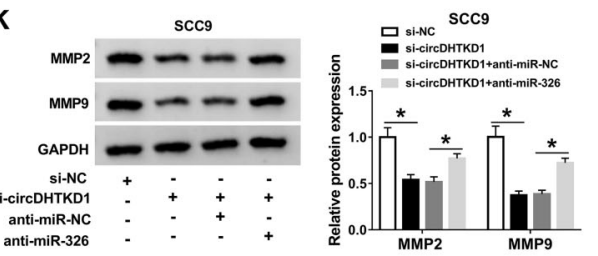

Figure 4. Inhibition of miR-326 partly restored the cancer-inhibitory function of downregulated circDHTKD1 in oral squamous cell carcinoma (OSCC) cells. A and B, The miR-326 level was measured through qRT-PCR after transfection of si-NC (negative control), si-circDHTKD1, si-circDHTKD1 + anti-miR-NC, and si-circDHTKD1 + anti-miR-326. Cell viability (C and D) and clonal capacity (E) were evaluated using MTT and colony formation assay. (F) The detection of cell apoptosis was completed via flow cytometry. Cell metastasis was assessed by wound healing and transwell assay for migration (G-I), as well as transwell assay and western blot for invasion (J-L). Three biological and three technical replicates were performed in each assay. Data are reported as means $\pm S D$. ${ }^{*} P<0.05$ (one-way ANOVA followed by Tukey's test).

circDHTKD1 might be used as a therapeutic target for OSCC. Overall, circDHTKD1 could sponge miR-326 to upregulate $\mathrm{GAB} 1$ expression to promote tumor growth and metastasis in OSCC (Figure 7G).

\section{Discussion}

circDHTKD1 is a novel circRNA with little exploration in cancer research. Fortunately, we have affirmed the carcinogenic action of circDHTKD1 in OSCC and its functional mechanism based on the miR-326/GAB1 axis in the current report, representing a comprehensive analysis of circDHTKD1 in OSCC.

Mounting evidence shows the abundant enrichment of circRNAs in human transcriptome (23). Recent studies have largely focused on the regulatory roles of circRNAs in various types of cancers. For example, circ_0016788 was revealed as a biological target for cancer progression and poor prognosis in hepatocellular carcinoma patients
(24), and circENTPD7 enhanced the motility and proliferation of glioblastoma cells (25). We also found a high level of circDHTKD1 in human OSCC tissues and cells. In addition, circDHTKD1 showed circRNA properties with high stability and cytoplasmic localization in OSCC cells. As for the functions of circRNAs in squamous cell carcinoma, knockdown of circUBAP2 hampers the malignant tumor behaviors in esophageal squamous cell carcinoma (ESCC) cells (26); circ_081069 contributes to cellular proliferative and migratory abilities in tongue squamous cell carcinoma (TSCC) (27); and circ_0011946 accelerates cell growth and metastasis of OSCC (28). Our assays indicated that circDHTKD1 knockdown reduced cell growth and promoted cell apoptosis. Due to the high metastatic ability of OSCC, we further explored the effect of circDHTKD1 on cell metastasis. Through the conduction of the wound healing assay, transwell assay, and protein detection, we found that downregulation of circDHTKD1 triggered the obstruction of metastasis in 
A

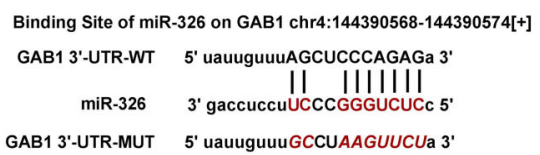

D
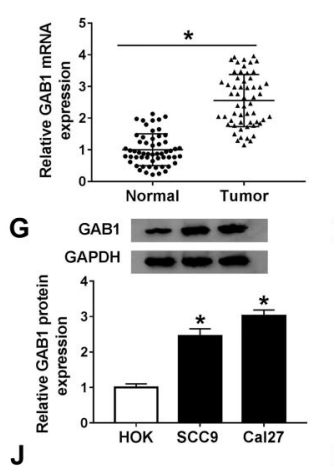

$\mathbf{J}$

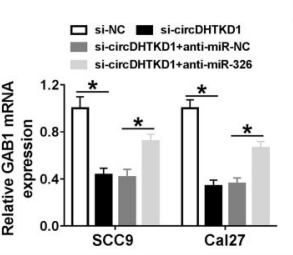

E

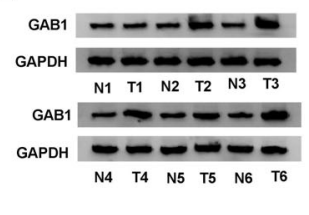

H

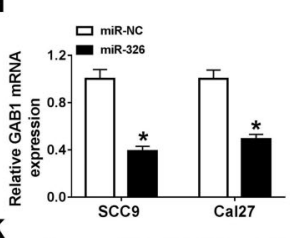

$\mathbf{K}$

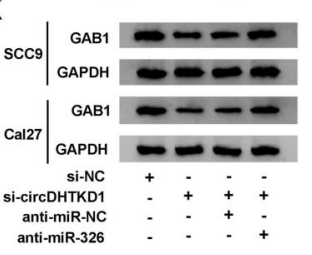

I
B
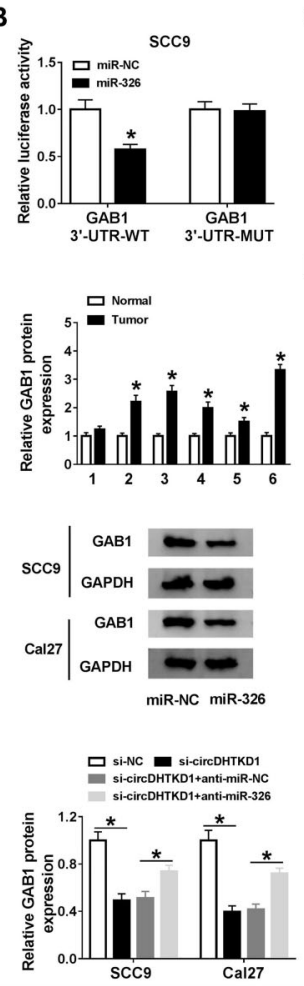

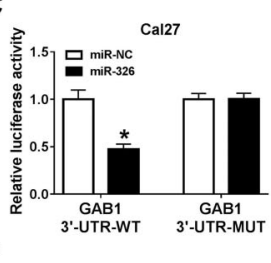

F
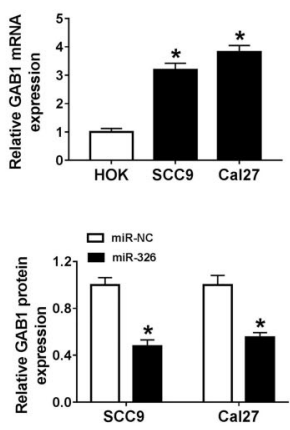

Figure 5. circDHTKD1 regulated GAB1 expression via interacting with miR-326. A, starBase was adopted to predict the binging region between miR-326 and GAB1 3'-UTR. B and C, Dual-luciferase reporter assay was used to determine whether miR-326 could combine with GAB1 3'-UTR in SCC9 and Cal27 cells. GAB1 mRNA and protein levels in OSCC tissues (D and E) and cells (F and G) were detected with qRT-PCR and western blot. qRT-PCR and western blot were used for assaying the mRNA and protein expression of GAB1 after transfection of miR-NC/miR-326 (H and $\mathbf{I})$ and si-circDHTKD1, si-circDHTKD1 + anti-miR-326, or the relative control groups $(\mathbf{J}$ and $\mathbf{K})$. Three biological and three technical replicates were performed in each assay. Data are reported as means $\pm S D$. ${ }^{*} P<0.05$ (Student's $t$-test and one-way ANOVA followed by Tukey's test).

OSCC cells. circDHTKD1 expression inhibition might be used as a targeted therapy for treating metastatic OSCC.

By using the online prediction software and Venn diagram analysis, we selected three miRNA targets for circDHTKD1 and finally affirmed that miR-326 was a definite target of circDHTKD1. circ_0001564 could regulate cell proliferation and apoptosis in osteosarcoma by playing the sponge role of miR-29c-3p (29), and circADAMTS13 reduced hepatocellular carcinoma cell proliferation by sponging miR-484 (30). Our reversed experiment exhibited that miR-326 downregulation mitigated the suppression of si-circDHTKD1 in the malignant progression of OSCC, suggesting that circDHTKD1 acted as an oncogenic factor in OSCC partly by sponging miR326. However, the reversal of miR-326 inhibitor for the function of si-circDHTKD1 was not complete in OSCC cells. Thus, the regulation of circDHTKD1 in OSCC was also associated with other factors. Other miRNA sponges remain to be discovered for circDHTKD1 in OSCC.

GAB1 $3^{\prime}$-UTR was found to be interplayed with miR326 , and miR-326 exerted a negative regulation on GAB1 expression in OSCC cells. GAB1 has been proven as a pro-cancer factor to promote proliferation in glioma (31), cell metastasis in breast cancer (32), and proliferation and invasion in non-small cell lung cancer (33). Herein, GAB1 also worked as an oncogene in OSCC to neutralize the miR-326-induced growth and metastasis inhibition, consistent with the previously published report of GAB1 in OSCC (17). Thus, OSCC progression was retarded by miR-326 that directly downregulated GAB1.

Through the sponge effect on miR-326, circDHTKD1 regulated GAB1 expression in a positive manner in OSCC cells. A good deal of circRNA/miRNA/mRNA regulatory networks have been unraveled in human cancers, like circAPLP2/miR-485-5p/FOXK1 in colorectal cancer (34) and circMYLK/miR-513a-5p/VEGFC in renal cell carcinoma (35). circDHTKD1/miR-326/GAB1 signal axis was first reported in OSCC in our current study. In the in vivo experiment, circDHTKD1 also facilitated tumorigenesis and invasion via modulating the miR-326/GAB1.

There are some limitations in the present study. Firstly, the reversed experiments of miR-326 and GAB1 for 
A

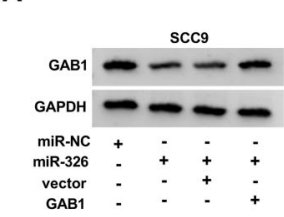

C

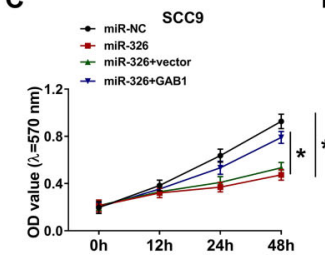

G

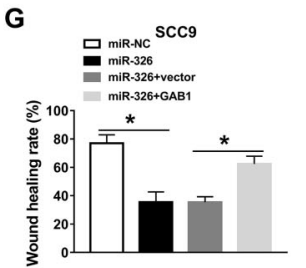

K

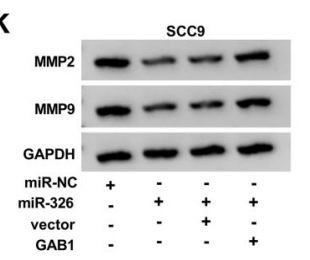

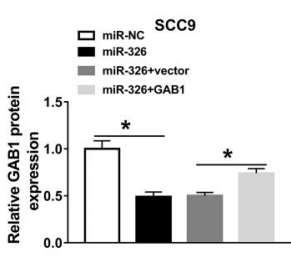

D

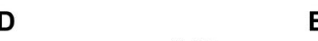

$-\operatorname{miR}-N C$
$-\operatorname{miR}-326$

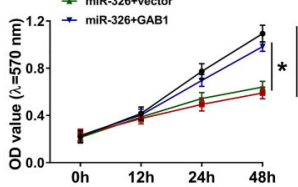

$\mathrm{H}$
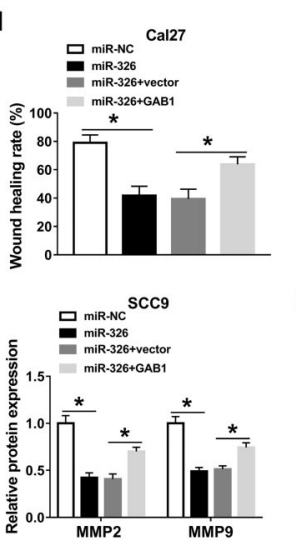

E

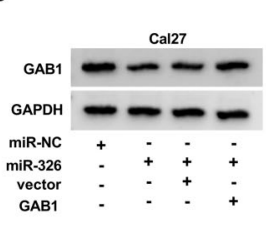

E

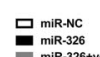

들 miR-323

B

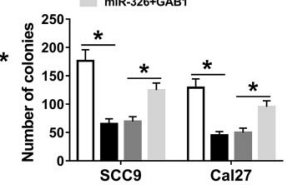

I

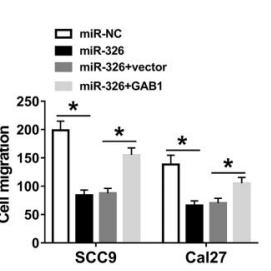

L

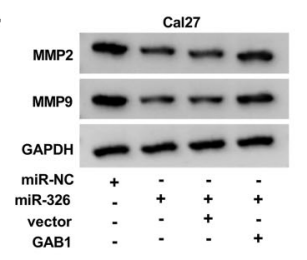

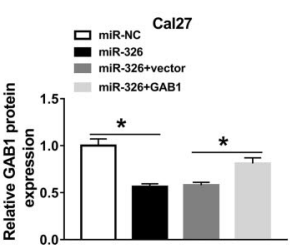

$\mathbf{F}$

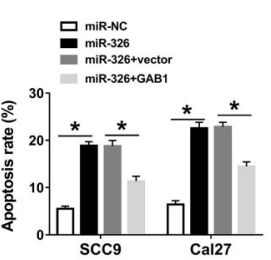

$\mathbf{J}$

믐 miR-Ne

mir-326+vector
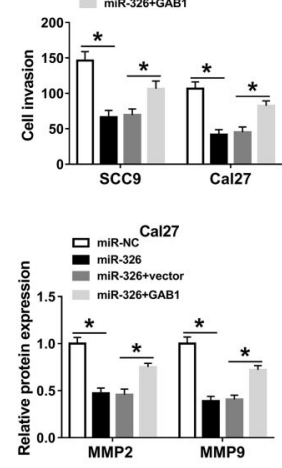

Figure 6. GAB1 downregulation was accountable for miR-326 as a tumor inhibitor in oral squamous cell carcinoma (OSCC). A and B, GAB1 protein analysis was conducted by western blot in SCC9 and Cal27 cells transfected with miR-NC (negative control), miR-326, miR-326 + vector, and miR-326 + GAB1. Cell growth was determined by cell viability via MTT (C and D) and clonal capacity via colony formation assay (E). F, Flow cytometry was used for assessing apoptotic cells. Wound healing assay, transwell assay, and western blot were used to assess cell migration $(\mathbf{G}-\mathbf{I})$ and invasion $(\mathbf{J}-\mathbf{L})$. Three biological and three technical replicates were performed in each assay. Data are reported as means $\pm \mathrm{SD}$. ${ }^{*} \mathrm{P}<0.05$ (one-way ANOVA followed by Tukey's test).

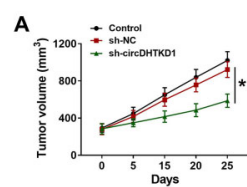

D

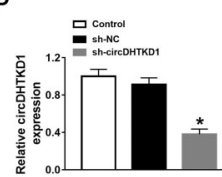

B
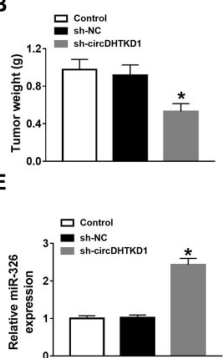

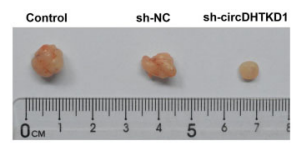

$\mathbf{F}$

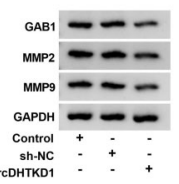

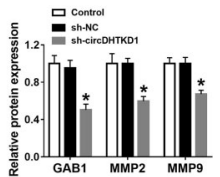

G

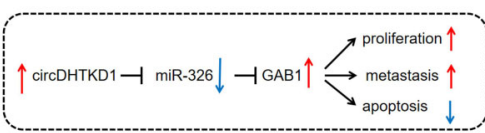

Figure 7. circDHTKD1 promoted oral squamous cell carcinoma (OSCC) progression in vivo by sponging miR-326 to regulate GAB1. A, Tumor volume was recorded after cell injection of control, sh-NC (negative control), and sh-circDHTKD1 groups every 5 days. Tumors were weighed after the excision from mice $(\mathbf{B})$ and tumor images were photographed $(\mathbf{C})$. D and $\mathbf{E}$, The measurement of circDHTKD1 and miR-326 in tumor tissues was performed using qRT-PCR. F, Western blot was employed to determine the levels of GAB1, MMP2, and MMP9 proteins. G, A schematic figure of the current study. Three biological and three technical replicates were performed in expression detection. Data are reported as means $\pm S D$. ${ }^{*} \mathrm{P}<0.05$ (one-way ANOVA followed by Tukey's test). 
circDHTKD1 in the in vivo assay should be performed to better affirm the conclusion of circDHTKD1/miR-326/ GAB1 axis in the regulation of OSCC. Secondly, miR-326 inhibitor did not reverse all those effects of si-circDHTKD1 on OSCC cells. The sponge function of circDHTKD1 on other miRNAs in OSCC progression remains to be explored. RNA sequencing after knockdown of circDHTKD1 may be a good manner to identify other potential miRNAs mediated by circDHTKD1. Thirdly, other downstream targets for miR-326 in OSCC should be explored. The different miRNA/mRNA axes will contribute to a better understanding of the functional mechanism of circDHTKD1 in OSCC.

\section{References}

1. D'souza S, Addepalli V. Preventive measures in oral cancer: an overview. Biomed Pharmacother 2018; 107: 72-80, doi: 10.1016/j.biopha.2018.07.114.

2. Chai AWY, Lim KP, Cheong SC. Translational genomics and recent advances in oral squamous cell carcinoma. Semin Cancer Biol 2020; 61: 71-83, doi: 10.1016/j.semcancer. 2019.09.011.

3. Michikawa C, Izumo $T$, Sumino J, Morita $T$, Ohyama $Y$, Michi $Y$, et al. Small size of metastatic lymph nodes with extracapsular spread greatly impacts treatment outcomes in oral squamous cell carcinoma patients. Int $J$ Oral Maxillofac Surg 2018; 47: 830-835, doi: 10.1016/j.ijom.2017.12.007.

4. Vatsyayan A, Mandlik D, Patel P, Patel P, Sharma N, Joshipura A, et al. Metastasis of squamous cell carcinoma of the head and neck to the thyroid: a single institution's experience with a review of relevant publications. $\mathrm{Br} \mathrm{J}$ Oral Maxillofac Surg 2019; 57: 609-615, doi: 10.1016/j.bjoms. 2019.05.012.

5. Aires FT, Lin CS, Matos LL, Kulcsar MAV, Cernea CR. Risk factors for distant metastasis in patients with oral cavity squamous cell carcinoma undergoing surgical treatment. ORL J Otorhinolaryngol Relat Spec 2017; 79: 347-355, doi: 10.1159/000485627.

6. Momen-Heravi F, Bala S. Emerging role of non-coding RNA in oral cancer. Cell Signal 2018; 42: 134-143, doi: 10.1016/ j.cellsig.2017.10.009.

7. Min A, Zhu C, Peng S, Rajthala S, Costea DE, Sapkota D. MicroRNAs as important players and biomarkers in oral carcinogenesis. Biomed Res Int 2015; 2015: 186904, doi: $10.1155 / 2015 / 186904$.

8. Ebbesen KK, Hansen TB, Kjems J. Insights into circular RNA biology. RNA Biol 2017; 14: 1035-1045, doi: 10.1080/ 15476286.2016.1271524.

9. Zou FW, Yang SZ, Li WY, Liu CY, Liu XH, Hu CH, et al. circRNA_001275 upregulates Wnt7a expression by competitively sponging miR3703p to promote cisplatin resistance in esophageal cancer. Int $J$ Oncol 2020; 57: 151-160, doi: $10.3892 /$ ijo.2020.5137.

10. Hu J, Ji C, Hua K, Wang X, Deng X, Li J, et al. Hsa_circ 0091074 regulates TAZ expression via microRNA1297 in triple negative breast cancer cells. Int $J$ Oncol 2020; 56: 1314-1326, doi: 10.3892/ijo.2020.5000.
In conclusion, circDHTKD1 increased the expression of GAB1 by sponging miR-326 in OSCC, inducing the promotion of tumor growth and metastasis in vitro and in vivo. This study developed a novel notion underplaying the OSCC carcinogenic mechanism at the molecular level by discovering the circDHTKD1/miR-326/GAB1 axis. circDHTKD1 may improve the diagnosis and treatment of OSCC as a potential biomarker.

\section{Acknowledgments}

The project was funded by Natural Science Fund Project of Hainan Province (No. 819MS125).

11. Yang J, Li Y, Yu Z, Zhou Y, Tu J, Lou J, et al. Circular RNA Circ100084 functions as sponge of miR23a5p to regulate IGF2 expression in hepatocellular carcinoma. Mol Med Rep 2020; 21: 2395-2404, doi: 10.3892/mmr.2020.11069.

12. Gao L, Zhao C, Li S, Dou Z, Wang Q, Liu J, et al. circ-PKD2 inhibits carcinogenesis via the miR-204-3p/APC2 axis in oral squamous cell carcinoma. Mol Carcinog 2019; 58: 1783-1794, doi: 10.1002/mc.23065

13. Zhu $X$, Shao $P$, Tang $Y$, Shu M, Hu WW, Zhang $Y$. hsa circRNA_100533 regulates GNAS by sponging hsa_ miR_933 to prevent oral squamous cell carcinoma. J Cell Biochem 2019; 120: 19159-19171, doi: 10.1002/jcb.29245.

14. He T, Li X, Xie D, Tian L. Overexpressed circPVT1 in oral squamous cell carcinoma promotes proliferation by serving as a miRNA sponge. Mol Med Rep 2019; 20: 3509-3518, doi: $10.3892 / \mathrm{mmr} .2019 .10615$.

15. Ouyang SB, Wang J, Zhao SY, Zhang XH, Liao L. CircRNA 0109291 regulates cell growth and migration in oral squamous cell carcinoma and its clinical significance. Iran J Basic Med Sci 2018; 21: 1186-1191, doi: 10.22038/ IJBMS.2018.30347.7313.

16. Tao D, Zhang Z, Liu X, Zhang Z, Fu Y, Zhang $P$, et al. LncRNA HOTAIR promotes the invasion and metastasis of oral squamous cell carcinoma through metastasisassociated gene 2. Mol Carcinog 2020; 59: 353-364, doi: 10.1002/mc.23159.

17. Xu L, Li J, Kuang Z, Kuang Y, Wu H. Knockdown of Gab1 inhibits cellular proliferation, migration, and invasion in human oral squamous carcinoma cells. Oncol Res 2018; 26: 617-624, doi: 10.3727/096504017X15043589260618.

18. Feng S, Liu N, Chen X, Liu Y, An J. Long non-coding RNA NEAT1/miR-338-3p axis impedes the progression of acute myeloid leukemia via regulating CREBRF. Cancer Cell Int 2020; 20: 112, doi: 10.1186/s12935-020-01182-2.

19. Pan G, Mao A, Liu J, Lu J, Ding J, Liu W. Circular RNA hsa_circ_0061825 (circ-TFF1) contributes to breast cancer progression through targeting miR-326/TFF1 signalling. Cell Prolif 2020; 53: e12720, doi: 10.1111/cpr.12720.

20. Salzman J, Gawad C, Wang PL, Lacayo N, Brown PO. Circular RNAs are the predominant transcript isoform from hundreds of human genes in diverse cell types. PLoS One 2012; 7: e30733, doi: 10.1371/journal.pone.0030733. 
21. Kunte M, Desai K. The inhibitory effect of C-phycocyanin containing protein extract (C-PC Extract) on human matrix metalloproteinases (MMP-2 and MMP-9) in hepatocellular cancer cell line (HepG2). Protein J 2017; 36: 186-195, doi: 10.1007/s10930-017-9707-0.

22. Cancemi P, Buttacavoli M, Roz E, Feo S. Expression of Alpha-Enolase (ENO1), Myc Promoter-Binding Protein-1 (MBP-1) and Matrix Metalloproteinases (MMP-2 and MMP9) reflect the nature and aggressiveness of breast tumors. Int J Mol Sci 2019; 20: 3952, doi: 10.3390/ijms20163952.

23. Memczak S, Jens M, Elefsinioti A, Torti F, Krueger J, Rybak $A$, et al. Circular RNAs are a large class of animal RNAs with regulatory potency. Nature 2013; 495: 333-338, doi: 10.1038/nature11928.

24. Cheng F, Wang L, Zhang J. Circular RNA 0016788 displays as a biomarker for tumor progression and poor prognosis in surgical hepatocellular carcinoma patients. J Clin Lab Anal 2020; 34: e23300, doi: 10.1002/jcla.23300.

25. Zhu F, Cheng $C$, Qin $H$, Wang $H$, Yu H. A novel circular RNA circENTPD7 contributes to glioblastoma progression by targeting ROS1. Cancer Cell Int 2020; 20: 118, doi: 10.1186/ s12935-020-01208-9.

26. Wu Y, Zhi L, Zhao Y, Yang L, Cai F. Knockdown of circular RNA UBAP2 inhibits the malignant behaviours of esophageal squamous cell carcinoma by microRNA-422a/Rab10 axis. Clin Exp Pharmacol Physiol 2020; 47: 1283-1290, doi: 10.1111/1440-1681.13269.

27. Wei T, Ye P, Yu GY, Zhang ZY. Circular RNA expression profiling identifies specific circular RNAs in tongue squamous cell carcinoma. Mol Med Rep 2020; 21: 1727-1738, doi: 10.3892/mmr.2020.10980.

28. Meng Y, Zhao EY, Zhou Y, Qiang DX, Wang S, Shi L, et al. Circular RNA hsa_circ_0011946 promotes cell growth, migration, and invasion of oral squamous cell carcinoma by upregulating PCNA. Eur Rev Med Pharmacol Sci 2020; 24: 1226-1232, doi: 10.26355/eurrev_202002_20175.

29. Song YZ, Li JF. Circular RNA hsa_circ_0001564 regulates osteosarcoma proliferation and apoptosis by acting miRNA sponge. Biochem Biophys Res Commun 2018; 495: 23692375, doi: 10.1016/j.bbrc.2017.12.050.

30. Qiu L, Huang Y, Li Z, Dong X, Chen G, Xu H, et al. Circular RNA profiling identifies circADAMTS13 as a miR-484 sponge which suppresses cell proliferation in hepatocellular carcinoma. Mol Oncol 2019; 13: 441-455, doi: 10.1002/ 1878-0261.12424.

31. Shao NY, Wang DX, Wang Y, Li Y, Zhang ZQ, Jiang Q, et al. MicroRNA-29a-3p downregulation causes Gab1 upregulation to promote glioma cell proliferation. Cell Physiol Biochem 2018; 48: 450-460, doi: 10.1159/000491776.

32. Xu BB, Gu ZF, Ma M, Wang JY, Wang HN. MicroRNA-590$5 p$ suppresses the proliferation and invasion of non-small cell lung cancer by regulating GAB1. Eur Rev Med Pharmacol Sci 2018; 22: 5954-5963, doi: 10.26355/eurrev_2018 $09 \_15926$.

33. Wāng X, Peng J, Yang Z, Zhou PJ, An N, Wei L, et al. Elevated expression of Gab1 promotes breast cancer metastasis by dissociating the PAR complex. J Exp Clin Cancer Res 2019; 38: 27, doi: 10.1186/s13046-019-1025-2.

34. Liu J, Zhang J, Wang Z, Xi J, Bai L, Zhang Y. Knockdown of circAPLP2 Inhibits Progression of Colorectal Cancer by Regulating miR-485-5p/FOXK1 Axis. Cancer Biother Radiopharm 2020, doi: 10.1089/cbr.2019.3310.

35. Li J, Huang C, Zou Y, Yu J, Gui Y. Circular RNA MYLK promotes tumour growth and metastasis via modulating miR513a-5p/NEGFC signalling in renal cell carcinoma. J Cell Mol Med 2020; 24: 6609-6621, doi: 10.1111/jcmm.15308. 